Document downloaded from:

http://hdl.handle.net/10251/62944

This paper must be cited as:

Doménech Antich, EM.; Jiménez Belenguer, Al.; Perez, R.; Ferrús Pérez, MA.; Escriche Roberto, MI. (2015). Risk characterization of antimicrobial resistance of Salmonella in meat products. Food Control. 57:18-23. doi:10.1016/j.foodcont.2015.04.001.

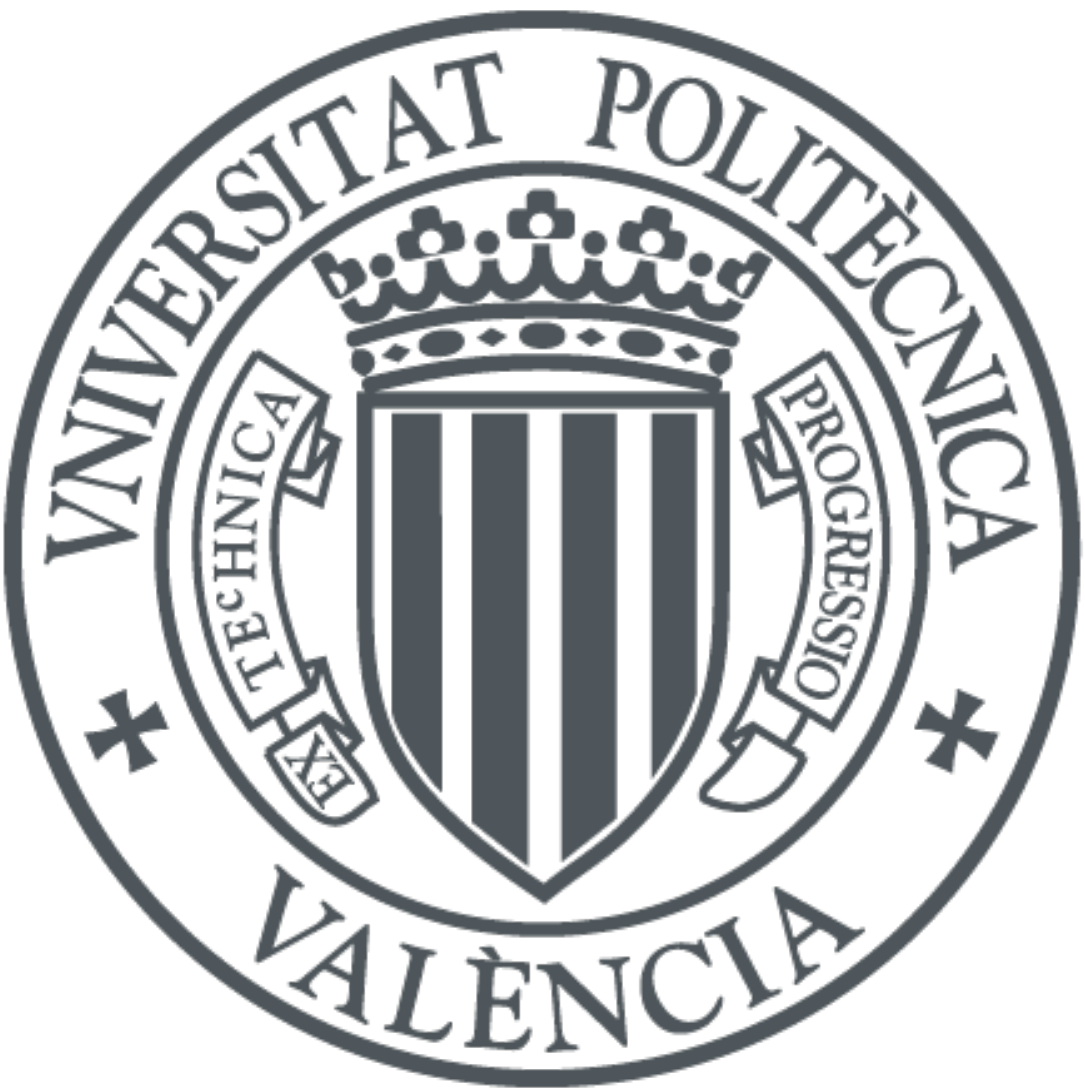

The final publication is available at

http://dx.doi.org/10.1016/j.foodcont.2015.04.001

Copyright Elsevier

Additional Information 


\title{
Risk characterization of antimicrobial resistance of Salmonella in meat products
}

\author{
Eva Doménech; Ana Jiménez-Belenguer; Rosa Pérez; Maria Antonia Ferrús; Isabel
}

Escriche

Food control 57 (2015) 18-23

\begin{abstract}
Antimicrobial resistance is considered to be a major public health concern in the framework of Horizon 2020. The risk to human health from foodborne antimicrobial resistant microorganisms can be determined and assessed using risk analysis tools in accordance with Codex principles. Therefore, the objective of this work was to determine the qualitative risk characterization of the presence of Salmonella resistant strains in meat products. Consequently, a total of 2050 whole and minced samples of poultry, pork and beef, were assessed. Samples were obtained by the Official Food Control Services of the Valencian administration (Spain), in the province of Valencia between January 2006 and June 2012. Salmonella was not found in any samples of beef. Salmonella strains isolated in poultry and pork samples were not resistant to amikacin, amoxicillin, cephalothin ciprofloxacin levofloxacin and ofloxacin. However, Salmonella isolates found in poultry had a high level of resistance to nalidixic acid, while those found in pork were more resistant to tetracycline and ampicillin. Furthermore, $41 \%$ of Salmonella isolates were resistant to three or more antibiotics. Finally, considering these results as exposure and taking into account the severity of the potential adverse health effects related to the different antimicrobials, risk characterization was estimated. As a result, three cases were classified as "Very high additional risk" all of them in minced meat, two cases in poultry (gentamicin and nalidixic acid) and one in pork (ampicillin). In the rest of the cases of resistance, the risk was classified as "high additional risk". This highlights the importance of continued surveillance and the need to take measures in the primary sector in order to minimize the risk for the consumer.
\end{abstract}




\section{Introduction}

Salmonella has long been recognised as an important zoonotic pathogen, which affects both animals and humans. This pathogen is most often detected in meat and meat products and constitutes one of the most common disease agents, which produces outbreaks of human illness due to the consumption of contaminated food (EFSA \& ECDC, 2014). Fortunately, the number of cases had a statistically significant decrease during the period 20062010 in Europe (EFSA/ECDC, 2012). Human salmonellosis is usually characterized by the acute onset of fever, abdominal pain, nausea, and sometimes vomiting after an incubation period of 12-36 hours. Symptoms are often mild and most infections are self-limiting, lasting a few days. However, in some patients, the infection may be more serious and the associated dehydration can be life threatening.

The common reservoir of Salmonella is the intestinal tract of animals. During slaughtering or processing, meat can become contaminated with faeces, and subsequently the food chain can act as a vector for the transfer of this bacterium. This is why the highest levels of non-compliance with Salmonella criteria generally occur in foods of meat origin (EFSA/ECDC, 2012). Between 2008 and 2010, minced meat and meat preparations from poultry, intended to be eaten cooked, had the highest level of non-compliance followed by minced meat and meat preparations from other animal species intended to be eaten cooked (EFSA/ECDC, 2012).

Infections are not the only problem. The incorrect use of antimicrobial agents, used in therapeutic treatments, promotes the development and spread of antimicrobialresistant bacteria in animals and also in humans via the food chain. (Thai et al., 2012; Maka, 2014; Doménech et al., 2015). Nowadays, antimicrobial resistance (AMR) is considered one of the main challenges to public health in the XXI century. In the European Union these resistances reach $26.4 \%$ of the confirmed human salmonellosis cases reported in the EU in
2011 (EFSA/ECDC, 2013). When pathogens become resistant to antimicrobial agents they can pose a greater risk to human health as a result of potential treatment failure, loss of treatment options and increased likelihood and severity of disease. In fact, treatment failures by multiresistant strains are responsible for half of the approximately 27,000 annual deaths from infections in the European Union (Watson, 2008). Furthermore, the economic burden on health systems is enormous. For example, the overall societal cost of infections due to antibiotic-resistant bacteria is estimated at about EUR 1.5 billion each year (ECDC/EMEA, 2009).

According to the European Commission, a co-ordinated research effort is required to bring more information related to resistance rates. With this aim in mind, one of the main goals of Horizon 2020 "Health, Demographic Change and Wellbeing", is the reduction of antibiotic resistance in the food chain. In accordance with Codex principles, risk analysis is an essential tool in assessing the risk to human health from foodborne AMR microorganisms and determining appropriate risk management strategies to control these risks. Over the past decade, there have been significant developments with respect to the use of risk analysis approaches in addressing antimicrobial resistance. Therefore, the objective of this work was to determine the qualitative risk characterization of the presence of Salmonella spp. resistant strains in poultry, pork and beef products analyzed by the Official Food Control Services of the Valencian administration (Spain) between January 2006 and June 2012.

\section{Material and methods \\ 2.1. Sample collection}

Sampling was carried out by the Official Food Control Services of the Department of Health of the Valencian administration between January 2006 and June 2012 with the aim of estimating the prevalence and antimicrobial resistance of Salmonella in fresh and minced poultry, pork and beef.

A total of 2050 samples were analyzed for the presence of Salmonella [fresh poultry (234), pork (196), beef (29); and minced 
poultry (151), pork (1270) and beef (170)]. The number of samples, the place where these were taken $(95 \%$ industry and $5 \%$ retail) and the type of food product to be analyzed were determined by the Valencian Health Administration as part of the Official Control process. Two main aspects were considered, on one hand Regulation (EC) $882 / 2004$ and on the other hand, information such as: consumption, the risk related to the product, data from the Rapid Alert System for Food and Feed, and data collected in previous years.

The transport time was no more than one hour in any case. A record of the name of the company, the batch, expiry date, date of manufacture, storage conditions, etc., was generated in each case.

\subsection{Sample examination}

Samples were examined by Public Health laboratory of Valencia, which is accredited following the standard ISO/IEC 17025:2005. The detection of pathogens was performed following the official method for Salmonella spp. (ISO 6579:2002).

\subsection{Antimicrobial susceptibility test}

Antimicrobial susceptibility testing of Salmonella was performed by the disk diffusion method according to the Clinical and Laboratory Standards Institute guidelines (CLSI, 2012). Susceptibility to fourteen antibiotics (Oxoid), including those used to treat human salmonellosis, was determined: amikacin (AK: $30 \mu \mathrm{g}$ ),

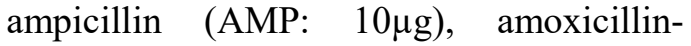
clavulanate (AMC: 20/10 $\mu \mathrm{g}$ ), sulfamethoxazole-trimethoprim (STX: $1.25 / 23.75 \mu \mathrm{g}$ ), ceftriaxone (CRO: $30 \mu \mathrm{g}$ ), ciprofloxacin (CIP: $5 \mu \mathrm{g}$ ), chloramphenicol

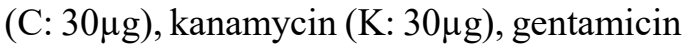
$(\mathrm{CN}: 10 \mu \mathrm{g})$, nalidixic acid (NAL: $30 \mu \mathrm{g}$ ), tetracycline (TE: $30 \mu \mathrm{g}$ ), cephalothin (KF: $30 \mu \mathrm{g}$ ), levofloxacin (LEV: $32-0.002 \mu \mathrm{g} / \mathrm{ml}$ ) and ofloxacin (O: $32-0.002 \mu \mathrm{g} / \mathrm{ml})$. E. coli ATCC 25922 and Enterococcus faecalis ATCC 29212 were used as control strains. The diameters of growth inhibition zones were measured and interpreted according to the breakpoints recommended by the CLSI for the various types of antibiotics, and the strains were classified as sensitive, intermediate (reduced susceptibility) or resistant. The resistant strains were confirmed by determining the Minimum Inhibitory Concentrations (MIC) by gradedconcentration antibiotic strips (E-test $\mathrm{AB}$ Biodisk), the interpretation of the results was carried out according to CLSI guidelines.

\subsection{Exposure assessment and hazard characterization}

In this paper the risk characterization uses the qualitative approach that estimates the level of exposure (presence of resistant Salmonella strains in meat and meat products) and characterizes the hazard (severity of the potential adverse health effects), table 1.

The exposure assessment was estimated using the percentage of antimicrobial resistance shown in the isolated strains. Then, based on each percentage one of the categories proposed by the European Food Safety Authority criteria (EFSA/ECDC, 2013) was assigned, as follows: "Extremely high (6)", >70\%; "Very high (5)", 50-70\%; "High (4)", 20-50\%; "Moderate (3)", 1020\%; "Low (2)", 1-10\%; "Very low (1)", $0.1-1 \%$; and "Rare (0)", $<0.1 \%$.

The hazard characterization was divided into four categories following the classification proposed by the WHO Advisory Group on Integrated Surveillance of Antimicrobial Resistance (WHO, 2012): "Negligible (0)", no adverse human health consequences or within normal limits; "Important (1)", important for human medicine; "Highly important (2)", highly important for human medicine; and "Critically important (3)", Critically important for human medicine.

The resulting risk characterization score can then be translated into meaningful qualitative risk categories, defined by the Codex in 2011. In this example, the product of the exposure assessment and hazard characterization are assigned to the following categories of risk:

- No Additional Risk: Value of 0

- Some Additional Risk: Value between 1 and 4

- High Additional Risk: Value between 5 and 10

- Very High Additional Risk: Value between 11 and 18 


\subsection{Statistical analysis}

Descriptive analyses of the data were undertaken using Statgraphics Centurion XVI.II (Statpoint Technologies, Inc. Warrenton, Virginia). Relative proportions were compared using the Chi-squared test $\left(\mathrm{X}^{2}\right)$ and Fisher's exact test. Also, comparisons of means were made. A probability value of less than $5 \%$ was deemed to be significant.

\section{Results}

\subsection{Prevalence of Salmonella serovars in meat products}

Overall Salmonella strains were isolated in $3.6 \%$ (74 out of 2050 samples) of the meat and minced meat products. The pork samples were the most contaminated by this microorganism $(4.5 \%)$, followed by poultry $(1.7 \%)$. It should be noted that no positive cases were found for beef, Table 2.

Seven serovars were identified in the resistant Salmonella strains. Typhimurium $(60.9 \%)$ was the most common serovar, followed by Enteritidis and Rissen, both with $14.6 \%$. Each of the serovars: Bredeney, Derby, Makumira and Virchow accounted for $2.4 \%$ of the total, Table 2. Focusing on the type of meat, Enteritidis serovar was more common in resistant strains isolated from poultry and Typhimurium was the most frequent serovar isolated from pork. The statistical study demonstrated that this relationship between the serotype and the animal species was significant ( $p$-value 0.0000).

\subsection{Antibiotic resistance}

Resistance to at least one antibiotic agent was found in $55.4 \%$ of isolates (41 out 74 positive strains). Salmonella strains isolated from minced poultry displayed the highest percentage of antibiotic resistance $(75 \%)$ closely followed by minced pork (61.4\%).

The resistance of Salmonella strains (isolated in samples of meat products) to 14 antibiotics was studied. No isolate was resistant to amikacin, amoxicillin/clavulanic ac, cephalothin, ciprofloxacin, levofloxacin, and ofloxacin. On the contrary, the highest level of resistance in Salmonella isolates was observed for tetracycline $39.8 \% \quad(\mathrm{n}=35$ strains), followed by ampicillin 20.5\% $(\mathrm{n}=18$ strains). For chloramphenicol, nalidixic acid and trimethoprim/sulphametoxazol the percentage of Salmonella resistance was $11.4 \%(\mathrm{n}=10$ strains $)$ in all cases. Salmonella had low resistance to gentamicin $3.4 \%(\mathrm{n}=3$ strains). It also had low resistance to ceftriaxone and kanamycin (1.1\%), but with only one strain resistant to each antibiotic. Moreover, possible relationships between antibiotic resistance and serotypes were studied; however no significant differences were found (p-value 0.7263).

It should be highlighted that Salmonella strains isolated from minced pork displayed the widest spectrum of antibiotic resistance, eight out of 14 analyzed. In addition, tetracycline was the antibiotic with the highest percentage of resistant Salmonella isolated in minced and fresh pork. For poultry, the highest spectrum of resistance was also found in minced meat, 5 out of 14 , and the most frequent antibiotic resistance, for fresh and minced poultry, was to nalidixic acid, figure 1 .

Table 3 shows the multiple antibiotic resistance pattern obtained for Salmonella and the involved meat products. A total of 17 out 74 strains $(22.9 \%)$ of all Salmonella isolates showed multidrug resistance. Tectracycline was present in all multiresistance patterns, followed by ampicillin, which was present in $82.4 \%$ of the cases. chloramphenicol (58.8\%) and trimethoprimsulfamethoxazole $(52.9 \%)$ were involved in more than $50 \%$ of the multi-resistance cases. Finally, nalidixic acid (29.4\%) and gentamicin $(11.8 \%)$ were the antibiotics least involved in multi-resistances.

In the present study, the most frequent serotype found in multiresistance strains was $S$. Typhimurium (75\%) followed by $S$. Rissen (12.5\%), $S$. Enteritidis and Bredeney, both with (6.25\%).

\subsection{Risk characterization}

Table 4 shows the results of risk characterization obtained from a combination of both components, i.e. hazard characterization and exposure assessment. 
Hazard characterization varies between 2 and 3. It is due to the fact that only antibiotics with important adverse human health consequences have been considered in this study. However, more differences were observed in the exposure taking values from 2 to 5. Results obtained for risk characterization highlight three cases as "Very high additional risk" all of them in minced meat, two cases in poultry (gentamicin and nalidixic acid) and one in pork (ampicillin). In the rest of the positive cases of resistance, the risk was classified as "high additional risk". However, the relationship between type of meat and type of antibiotic was not significant ( $p$ value 0.4507 and $p$ value 0.2807 , respectively).

\section{Discussion}

The highest levels of non-compliance with Salmonella criteria generally occur in foods of meat origin, which are intended to be cooked before consumption (EFSA/ECDC, 2014). Therefore, Salmonella contamination in these products has been widely investigated worldwide. Results show a great variation depending on the information source. As regards the conclusions presented by the EFSA/ECDC, (2014) for the European Union countries, Salmonella was most often detected in fresh broiler and turkey meat, being less often detected in pork or beef. Moreover, minced meat and meat preparations from poultry intended to be eaten cooked had the highest level of non-compliance, with percentages that varied from 0 to $48.5 \%$. However, the results found in the present paper for the incidence of Salmonella in poultry (2.6\%) are more similar to the values obtained by Wilson (2002) who found $2 \%$ in raw retail poultry and Soultos et al., (2003) with 3 positive samples out of $205(1.5 \%)$.

In relation to pork, our results were around $4.5 \%$ of samples contaminated with Salmonella. This value is higher than the $2.6 \%$ of Salmonella spp. recovered from pork samples by Prendergast in (2009). Nevertheless, our finding is in the interval of non-compliance among the European Union countries reported in 2012 for Salmonella for minced meat and meat preparations, from animal species other than poultry intended to be eaten cooked, which ranged from 0 to $6.1 \% \quad$ (EFSA/ECDC, 2014). This inconsistency of prevalence from different countries might be biased because of different stages where they sampled, varied compositions of sample types and detection methods, (Zhu et al., 2014).

Enteritidis and Typhimurium were the serovars which displayed highest patterns of resistance. These results are consistent with previous studies (EFSA/ECDC, 2013; Maka et al., 2014). Moreover, these serovars are the most frequently associated with human illness in the European Union. Human cases caused by $S$. Enteritidis are most commonly related to the consumption of contaminated eggs or poultry, whereas S. Typhimurium cases are mostly associated with the consumption of contaminated pork, beef or poultry (DANMAP, 2012; EFSA/ECDC, 2012; Hur et al., 2012). This association between serovars and products agrees with our results and with previous studies. Prendergast et al., (2009) showed that $S$. Typhimurium accounted for almost all of the serotypes $(85 \%)$ isolated from raw pork samples, as was the case for Salmonella recovered from pork cuts in the factory environment. $S$. Typhimurium has remained the dominant Salmonella serotype in Irish pork over the last 5 years (Boughton et al., 2004; Jordan et al., 2006) and it is also one of the most frequently isolated serotypes in humans in Ireland (Foley et al., 2007).

It should be highlighted that $S$. Typhimurium displayed resistance to the widest spectrum of antimicrobials ( 8 out 14 studied). Similar results were found by Maka et al., (2014), who concluded that $S$. Typhimurium displayed resistance to 10 of the 19 tested compounds. Resistance to the antimicrobials ampicillin and tetracyclines was frequent for $S$. Typhimurium isolates from pork and poultry and varied between 23.5\% and 83.1\% (Glenn et al. 2011; Bacci et al., 2012; Van Boxstael et al., 2012).

In the current study, resistance to amikacin, amoxicillin, cephalothin ciprofloxacin levofloxacin and ofloxacin was not found in any Salmonella strain. Taking into account the critical importance for human medicine of these antimicrobials (WHO, 2012), the obtained results are encouraging. On the contrary, the greatest resistance percentages were found for 
tetracycline $56 \%$ followed by ampicillin $30 \%$ in minced pork and nalidixic acid 50\% in minced poultry. These findings concur with results obtained by EFSA/ECDC, (2013), which concluded that Salmonella isolates, from meat and animals, were resistant to tetracyclines, ampicillin and sulfonamides at levels of $7 \%$ to $61 \%$. Moreover, resistance was higher in isolates from pigs and turkeys than in those from broilers, laying hens and cattle. Resistance to ciprofloxacin and nalidixic acid was higher in Salmonella isolates from broilers and turkeys $(33-50 \%)$ than it was in isolates from laying hens, pigs or cattle (1-13\%). Similar findings were found by Thai, et al., (2012) who indicated that nalidixic acid was reported as the predominant resistance in poultry and concurs with previous studies in Portugal (Antunes et al., 2003), Thailand (Padungtod and Kaneene, 2006), China (Yan et al., 2010) and Mexico (Miranda, et al., 2009). However, in a previous study, all tested Salmonella strains isolated from poultry in Spain were resistant to nalidixic acid (Álvarez-Fernández, et al., 2012).

In relation to multi-resistant isolates, resistance to tetracycline was present in all cases, followed by ampicillin in $82 \%$. In this case, both are antimicrobial agents used to treat serious human disease (WHO, 2012). However, fortunately, only the second one is considered an antimicrobial agent that is used to treat diseases caused by either: (1) organisms that may be transmitted to humans from non-human sources or, (2) human diseases caused by organisms that may acquire resistant genes from non-human sources. In both cases, multi-resistance can influence the severity of the illness and made its treatment difficult. This highlights the importance of continued surveillance and the usefulness of this information to take measures in the primary sector. However, more epidemiological data and resistance mechanisms are needed in order to be more accurate when the risk assessment is defined.

\section{Acknowledgments}

This study was performed in collaboration with the General Director of Public Health of the Valencian Government Health Department. However, the views expressed here do not necessarily reflect the positions or policies of this Health Department. The authors are grateful to all inspectors from the Public Health Department of the Valencia Regional Government who participated in the monitoring program and thank the technicians of the Public Health Laboratory of Valencia Regional Government.

\section{References}

Antunes, P., Réu, C., Sousa, J.C., Peixe, L., \& Pestana, N., (2003). Incidence of Salmonella from poultry products and their susceptibility to antimicrobial agents. International Journal of Food Microbiology, 82, 97-103.

Álvarez-Fernández, E., Alonso-Calleja, C., García-Fernández, C., \& Capita, R. (2012). Prevalence and antimicrobial resistance of Salmonella serotypes isolated from poultry in Spain: comparison between 1993 and 2006. International Journal of Food Microbiology, 153, 281-287.

Bacci, C., Boni, E., Alpigiani, I., Lanzoni, E., Bonardi, S., \& Brindani, F. (2012) Phenotypic and genotypic features of antibiotic resistance in Salmonella enterica isolated from chicken meat and chicken and quail carcasses. International Journal of Food Microbiology, 160, 16-23.

Boughton, C., Leonard, F.C., Egan, J., Kelly, G., O'Mahony, P., Markey, B.K., \& Griffin, M. (2004). Prevalence and number of Salmonella in Irish retail pork sausages. Journal of Food Protection 67, 1834-1839.

CLSI (2012). Performance Standards for Antimicrobial Disk Susceptibility Test; Approved Standard-Eleventh edition. CLSI document M02-A11. Wayne, PA: Clinical and Laboratory Standards Institute.

Codex (2011). Guidelines for risk analysis of foodborne antimicrobial resistance. Codex Alimentarius Commission/Guidelines CAC/GL 772011.

http://www.codexalimentarius.org/sta ndards/list-ofstandards/en/?provide=standards\&or derField=fullReference \&sort=asc \&n 
um $1=\mathrm{CAC} / \mathrm{GL}$ (Accessed November 2014).

DANMAP (2012). Use of antimicrobial agents and occurrence of antimicrobial resistance in bacteria from food animals, food and humans in Denmark.

http://www.danmap.org/Downloads/ $\sim /$ media/Projekt\%20sites/Danmap/D ANMAP\%20reports/DANMAP\%20 2012/Danmap_2012.ashx (Accessed November 2014).

Doménech, E., Jimenez-Belenguer, A., Amoros, J.A., Ferrus, M.A., \& Escriche, I. (2015). Prevalence and antimicrobial resistance of Listeria monocytogenes and Salmonella strains isolated in ready-to-eat foods in Eastern Spain. Food Control, 47, 120-125.

ECDC/EMEA (2009). The bacterial challenge: time to react.

http://www.ecdc.europa.eu/en/public ations/

Publications/0909_TER_The_Bacteri al_Challenge_Time_to_React.pdf (Accessed March 2014)

EFSA/ECDC (2012). The European Union Summary Report on Trends and Sources of Zoonoses, Zoonotic Agents and Food-borne Outbreaks in 2010. Journal; 10(3):2597. http://www.efsa.europa.eu/en/efsajou rnal/doc/2597.pdf.

(Accessed November 2014).

EFSA/ECDC (2013). The European Union Summary Report on antimicrobial resistance in zoonotic and indicator bacteria from humans, animals and food in 2011. EFSA Journal, 11(5):3196.

http://www.efsa.europa.eu/de/efsajou rnal/pub/3196.htm\#tabs-1 (Accessed March 2014).

EFSA/ECDC (2014). The European Union Summary Report on Trends and Sources of Zoonoses, Zoonotic Agents and Food-borne Outbreaks in 2012. EFSA Journal, 12(2):3547 http://www.efsa.europa.eu/en/efsajou rnal/pub/3547.htm (Accessed March 2014).

Foley, B., McKeown, P., de Lappe, N. \& Cormican, M., (2007). Salmonellosis in Ireland, 2006. Epi-Insight, 8, 2-3.
Glenn, L.M., Lindsey, R.L., Frank, J.F., Meinersmann, R.J., Englen, M.D., Fedorka-Cray, P.J., \& Frye, J.G. (2011). Analysis of antimicrobial resistance genes detected in multidrugresistant Salmonella enterica serovar Typhimurium isolated from food animals. Microbial Drug Resistance 17, 407-418.

Hur, J., Jawale, C., \& Lee, J.H. (2012). Antimicrobial resistance of Salmonella isolated from food animals: A review. Food Research International, 45, 819-830.

ISO 6579:2002. Microbiology of food and animal feeding stuffs - Horizontal method for the detection of Salmonella spp. ISO Standards, last revision 2012.

ISO/IEC 17025:2005. General requirements for the competence of testing and calibration laboratories. ISO Standards, last revision 2010.

Jordan, E., Egan, J., Dullea, C., Ward, J., McGillicuddy, K., Murray, G., Murphy, A., Bradshaw, B., Leonard, N., Rafter, P., \& McDowell, S. (2006). Salmonella surveillance in raw and cooked meat and meat products in the Republic of Ireland from 2002 to 2004. International Journal of Food Microbiology 112, 66-70.

Mąka, L., Maćkiw, E., Ścieżyńska, H., Pawłowska, K., \& Popowska, M. (2014). Antimicrobial susceptibility of Salmonella strains isolated from retail meat products in Poland between 2008 and 2012. Food Control 36, 199-204.

Miranda, J.M., Mondragon, A.C., Martinez, B., Guarddon, M., \& Rodriguez, J.A. (2009). Prevalence and antimicrobial resistance patterns of Salmonella from different raw foods in Mexico. Journal of Food Protection, 72, 966971.

Padungtod, P., \& Kaneene, J.B. (2006). Salmonella in food animals and humans in north Thailand. International Journal of Food Microbiology 108, 346-354.

Prendergast, D.M., Duggan, S.J., GonzalesBarron, U., Fanning, S. Butler, F. Cormican, M, \& Duffy, G. (2009). 
Prevalence, numbers and characteristics of Salmonella spp. on Irish retail pork. International Journal of Food Microbiology 131, 233-239.

Soultos, N., Koidis, P., \& Madden, R.H. (2003). Presence of Listeria and Salmonella spp. in retail chicken in Northern Ireland. Lett. Appl. Microbiol., 37, 421-423.

Thai, TH., Hirai, T., Lan, NT., \& Yamaguchi, R. (2012). Antibiotic resistance profiles of Salmonella serovars isolated from retail pork and chicken meat in North Vietnam. International Journal of Food Microbiology, 156, 147-151.

Van Boxstael, S., Dierick, K., Van Huffel, X., Uyttendael, M., Berkvens, D., Herman, L., Bertrand, S., Wildemauwe, C., Catry, B., Butaye, P., \& Imberechts, H. (2012). Comparison of antimicrobial resistance patterns and phage types of Salmonella Typhimurium isolated from pigs, pork and humans in Belgium between 2001 and 2006. Food Research International, 45, 913-918.

Watson, R. (2008). Multidrug resistance responsible for half of deaths from healthcare associated infections in Europe. Brit. Med Journal, 336, 12661267.

Wilson, I.G. (2002). Salmonella and Campylobacter contamination of raw retail chickens from different producers: a six-year survey. Epidemiol. Infect., 129, 635-645.

WHO (2012). Critically Important Antimicrobials for Human Medicine. 3rd Revision 2011. World Health Organization.

http://apps.who.int/iris/bitstream/106 65/77376/1/9789241504485_eng.pdf. (Accessed November 2014)

Yan, H., Li, L., Alam, M. J., Shinoda, S., Miyoshi, S.I., \& Shi, L. (2010). Prevalence and antimicrobial resistance of Salmonella in retail foods in northern China. International Journal of Food Microbiology, 143, 230-234.

Zhu, J., Wang, Y., Song, X., Cui, S., Xu, H., Yang, B., Huang, J., Liu, G., Chen, Q., Zhou, G., Chen, Q., Li, F. (2014).
Prevalence and quantification of Salmonella contamination in raw chicken carcasses at the retail in China. Food Control, 44, 198-202. 